\title{
Creatividad táctica y funciones ejecutivas en los deportes de interacción
}

\section{Tactical creativity and executive functions in interactive sports}

\author{
Francisco Alarcón López ${ }^{1}$, Alberto Castillo Díaz ${ }^{1}$, Nuria Ureña Ortín ${ }^{2}$, Elisa Torre Ramos ${ }^{3}$ y David Cárdenas Vélez ${ }^{3}$ \\ 1 Facultad de Deporte. Universidad Católica San Antonio de Murcia (Ucam) \\ 2 Facultad de Educación. Universidad de Murcia \\ 3 Facultad de Ciencias de la Actividad Física y el Deporte. Universidad de Granada
}

\begin{abstract}
Resumen: La creatividad táctica es una de las características de los jugadores con mayor pericia en deportes de interacción. Ésta se define como la capacidad para generar respuestas sorpresivas y originales ante los problemas que surgen durante el juego. Recientes investigaciones han comprobado que estos deportistas poseen ciertas capacidades cognitivas, conocidas como funciones ejecutivas, que podrían explicar esta superioridad. Estudios experimentales llevados a cabo con niños concluyen que la práctica repetida de actividad física en entornos con elevados niveles de entropía podría mejorar estas funciones ejecutivas. Por otro lado, se ha visto que el uso de estrategias intencionales por parte del entrenador para dirigir la conducta de los deportistas puede ser perjudicial para el desarrollo de la creatividad táctica. En esta revisión se describe esta evidencia y se realiza una propuesta práctica sobre cómo diseñar entornos de aprendizaje para promover esta capacidad a través del control de la complejidad de la tarea y del feedback del entrenador.
\end{abstract}

Palabras clave: incidental, incertidumbre, entropía, if-then, inteligencia.
Abstract: Tactical creativity is one of the characteristics of the players with greater expertise in interactive (or contact) sports. The term is defined as the ability to generate original and surprising answers to the problems that arise during the game. Recent research has shown that these athletes have certain cognitive capacities, known as executive functions, which could explain this superiority. Experimental studies conducted with children conclude that the repeated practice of physical activity in environments with high levels of entropy could improve these executive functions. On the other hand, it has been seen that the use of intentional strategies by the coach, meant to address the behavior of athletes, can be detrimental to the development of tactical creativity. This review describes the evidence and offers a practical proposal on the design of learning environments promoting this capacity through the control of the task complexity and the feedback of the coach. Key words: Incidental, uncertainty, entropy, if-then, intelligence.
Desde hace décadas, los científicos del deporte vienen estudiando los factores que pueden ser determinantes para el éxito o rendimiento deportivo. Recientemente, en el ámbito de la psicología se han encontrado evidencias sobre el valor predictivo de ciertas capacidades cognitivas. Así, cuando se comparan deportistas con diferentes niveles de pericia en tareas de un dominio específico, los expertos han demostrado ser superiores en tareas que implican el reconocimiento y recuerdo de patrones de comportamiento (Abernethy, Baker \& Côté, 2005; Smeeton, Ward \& Williams, 2004), estrategias de búsqueda visual (Vaeyens, Lenoir, Williams \& Philippaerts, 2007; Williams, 2000) y el conocimiento de las probabilidades situacionales (North \& Williams, 2008; Williams, Hodges, North \& Barton, 2006), es decir, el saber cuál es la probabilidad de que algo suceda en el contexto real deportivo para actuar en consecuencia.

Desde este enfoque, se han intentado averiguar las claves de lo que algunos autores han definido como inteligencia táctica, sentido o habilidad del juego, y cuyo objetivo principal es encontrar la solución ideal a los problemas derivados de la interacción en el entorno deportivo (Memmert \& Roth, 2007). Para ello el jugador debe generar reglas de acción (si-

Dirección para correspondencia [Correspodence address]: Alberto Castillo Díaz. Facultad de Deporte. Universidad Católica San Antonio de Murcia (Ucam).E-mail: acastillo@ucam.edu entonces; if-then) que relacionen su actuación eficaz con las claves atencionales que se encontraron presentes en el momento de la toma de decisión. Dicho de otro modo, la actuación del deportista dependerá del grado de cumplimiento de ciertas claves o de la relación entre ellas (Perales, Cárdenas, Piñar, Sánchez \& Courel, 2011).El aprendizaje de estas claves resulta determinante para reducir el nivel de incertidumbre al que está sometido el jugador, para predecir las acciones de los adversarios y, en consecuencia, para establecer conductas de anticipación. En este contexto destacan aquellos jugadores que poseen la habilidad para generar un elevado nivel de incertidumbre en el rival al dar una respuesta novedosa ante una situación de juego ya conocida. Esto es posible gracias a que las personas son capaces de aprender reglas complejas de manera intuitiva, y generar así principios de acción, lo que según Hogarth (2001) constituye la base del razonamiento abstracto. Ante una misma situación, el jugador deberá tener en cuenta más de uno de estos principios, siendo incluso posible que el cumplimiento de alguno de ellos se produzca de manera inversamente proporcional al de otros. Por ejemplo, a medida que el jugador atacante con balón se aleje del oponente, mayor será el nivel de protección del balón y la posibilidad de lanzar con más seguridad pero, al mismo tiempo, mayor la distancia a la meta, perdiendo eficacia (Cárdenas \&Alarcón, 2010). Inevitablemente, los jugadores deben elaborar su respuesta valorando constantemente esta relación costo-bene- 
ficio. El jugador creativo es aquel capaz de, ante una misma situación, modificar constantemente las prioridades de estos principios, mostrando una alta variabilidad en sus decisiones. La creatividad también ha sido identificada con la capacidad de pasar de forma muy eficaz (asistencia) en condiciones de juego muy complejas. Según Vestberg, Gustafson, Maurex, Ingvar y Petrovic (2012), estos jugadores poseen ciertas capacidades que son reconocidas con el término de "leer el juego". Estas circunstancias de juego vienen definidas por un nivel elevado de entropía, es decir de elevada variabilidad (Marek, Noworol \& Karkowski, 1988), una disminución del tiempo para procesar la información relevante (Cárdenas, Conde \& Perales, 2015), y la presencia tanto de estímulos simultáneos a los que atender como de información presente que inhibir (Romeas, Guldner \& Faubert, 2015).

De hecho, se ha comprobado que, en condiciones de laboratorio, los expertos consiguen mejores resultados en entornos complejos. Chaddock, Neider, Voss, Gaspar, y Kramer (2011) han encontrado diferencias significativas entre deportistas y no deportistas en la capacidad de analizar escenas que requieren la multitarea. Faubert (2013), utilizando el paradigma del "Multiple Object Tracking (MOT)", encontró que los jugadores profesionales de deportes de equipo tenían mayor capacidad de aprendizaje en una tarea de laboratorio que consistía en el seguimiento de objetos en movimiento en un entorno en el que existían distractores que debían evitar. Además, estudios recientes, como los de Mangine et al. (2014) o Romeas et al. (2015), avalan la transferencia de estas habilidades al campo. En el primero, se comprobó que los jugadores de la NBA mostraban una alta correlación entre su capacidad de ver y responder a estímulos en la cancha con su rendimiento en el MOT. En el segundo, los autores consiguieron mejoras en la capacidad de pasar en jugadores universitarios de fútbol tras un entrenamiento basado en el MOT. Estos autores sugieren que la capacidad de aprendizaje en contextos complejos, dinámicos e impredecibles es una de las imprescindibles para alcanzar la élite en estos deportes.

En esta línea, autores como Memmert y Furley han estudiado las limitaciones de jugadores de deportes de equipo para percibir compañeros que se desmarcan de manera inesperada. Memmert y Furley (2007) y posteriormente Furley, Memmert, y Heller (2010) pidieron a jugadores amateurs de baloncesto y balonmano que atendieran durante una secuencia de vídeo al jugador con balón y a su adversario directo, y que tras finalizar el vídeo eligieran la decisión táctica más idónea para conseguir encestar o marcar el gol. Los resultados obtenidos mostraron que solo el $55 \%$ de los jugadores en el primer estudio y el $62 \%$ en el segundo, fueron capaces de ver al jugador desmarcado. En estudios posteriores, cuando se ha aumentado la complejidad de la tarea, acercándose más a la realidad, estos porcentajes fueron aún menores (Alarcón \& Verdú, 2012).
Para reducir la incertidumbre, el jugador ante condiciones de tan alta complejidad, no podrá usar unas pocas reglas de decisión "si-entonces", sino un conjunto complejo de ellas (Cárdenas et al, 2015). Para conseguir un comportamiento adaptativo en estos entornos, en los que los mapas de estímulo-respuesta que se pueden producir van a ser débiles, variados, o van a cambiar con rapidez, será necesario recurrir a representaciones de metas y medios, y es ahí donde la corteza prefrontal (CPF) actúa como protagonista principal encargada de desarrollar esta función (Tirapu-Ustárroz, García-Molina, Luna-Lario, Verdejo-García \& Ríos-Lago, 2012). Para Lezak (1982) las funciones ejecutivas se presentan como las capacidades mentales esenciales para llevar a cabo una conducta eficaz y creativa. Según Miyake et al. (2000), estas funciones tienen tres componentes que, aunque claramente diferenciados, no son independientes, y contribuyen de manera diferencial al rendimiento en tareas de tipo ejecutivo. Estos componentes son Actualización: monitorización, manipulación y la actualización de la información en línea, de la memoria de trabajo; Inhibición: capacidad para inhibir de forma deliberada o controlada la producción de respuestas predominantes automáticas cuando la situación lo requiere; y Alternancia: capacidad de cambiar de manera flexible entre distintas operaciones mentales o esquemas.

En este punto es importante distinguir entre los conceptos de creatividad táctica e inteligencia táctica, los cuales se basan en la teórica distinción entre el pensamiento divergente y el convergente, respectivamente (Guilford, 1967; Sternberg \& Lubart, 1999; Runco, 2007). La inteligencia táctica correspondería al primer tipo de pensamiento, mientras que la creatividad es la expresión de un pensamiento divergente y que en los deportes se puede definir como la capacidad para generar respuestas distintas a las habituales en la resolución de los problemas que plantean las situaciones de juego, que pueden ser descritas como sorpresivas, raras y originales. Según Memmert (2014), las características que definirían las conductas creativas son: originalidad (lo inusual de la toma de decisiones en las acciones tácticas); flexibilidad (la variedad en la toma de decisiones tácticas está determinada por la diversidad de acción y respuestas de los jugadores); fluidez (el número de decisiones tácticas que los jugadores generan en función de la variabilidad situacional dada). Para Ben-Soussan, Berkovich-Ohana, Piervincenzi, Glicksohn, y Carducci, (2015), dos componentes esenciales de la creatividad son la fluidez y la flexibilidad cognitiva.

Hay estudios que han encontrado relaciones entre estas capacidades en jugadores de fútbol entrenados e indicadores de rendimiento durante la competición. Los jugadores que más goles y asistencias consiguieron en una temporada fueron los que mayores niveles de flexibilidad cognitiva mostraron. Además estas variables fueron predictoras de un rendimiento futuro dos temporadas más tarde (Vestberg et al., 2012). Por 
otra parte, estas funciones cognitivas de orden superior han sido relevantes para la identificación y el desarrollo del talento de jugadores de fútbol juvenil (Verburgh, Scherder, van Lange \& Oosterlaan, 2014). Es por ello que la creatividad táctica, al igual que la inteligencia táctica, es igualmente considerada determinante en el proceso de detección y desarrollo del talento (Williams, 2013).

No obstante, tal como apuntan Cárdenas y Torre (2005), en el contexto deportivo, formalmente reglado, las soluciones vienen condicionadas por la necesidad de obtener eficacia, lo que restringe parcialmente el comportamiento individual y distingue el acto motor de cualquier otra actividad donde poder manifestarse de forma creativa. Es por ello que la respuesta creativa en el contexto deportivo no pueda ser permanentemente original, novedosa pero puede estar asociada a la capacidad para generar respuestas impredecibles que sorprendan a los rivales.

No es casual que el mayor desarrollo de estas capacidades se produce en entornos con un elevado nivel de incertidumbre, de variabilidad contextual, de interacción entre miembros de equipos rivales. Alves et al. (2013) creen necesario analizar las condiciones cognitivas de los deportes de interacción para entender las mejoras que se producen desde el punto de vista cognitivo. Piensan que, al igual que se ha observado que los animales en un ambiente enriquecido cognitivamente consiguen mejoras sustanciales de su capacidad de aprendizaje y memoria(Park \&Shaw, 1992; Van Praag, Christie, Sejnowski \& Gage, 1999), en los deportes de interacción se podrían producir efectos similares gracias al elevado nivel de entropía de las tareas, lo que implica grandes desafíos físicos y mentales. Para Best (2010), cuando en el entorno existe una interferencia contextual, es decir, los componentes de la tarea se presentan de una manera compleja y cuasi-aleatoria, las habilidades que se aprenden, se retienen y se transfieren mejor. Esta interferencia contextual, típica de los deportes de interacción, demanda procesos ejecutivos como crear un plan de acción motriz, supervisado y modificado en presencia continua de demandas cambiantes de la tarea (Brady, 2008). Por lo tanto, es probable que el tratamiento de la información sea más elaborado, dando lugar a un mejor aprendizaje (Carey, Bhatt \& Nagpal, 2005). En un estudio reciente, el aprendizaje en condiciones de interferencia contextual exigía una mayor actividad de los circuitos y estructuras relacionadas con las FEs, generando así una mayor activación frontal, en comparación con un aprendizaje en ausencia de interferencia, cuya activación más generalizada fue parietal, premotora, y del cerebelo (Cross, Schmitt \& Grafton, 2007).

Autores como Ben-Soussan et al. (2015), Martín et al. (2014), Martins y Gotuzo (2015) y Pesce et al. (2013) han conseguido mejoras significativas de las FEs a través de la práctica de ejercicio físico que conlleva un compromiso cognitivo, gracias a la manipulación de la incertidumbre. Estos autores sugieren las bondades de los juegos reducidos como medio para la mejora de algunos de los componentes de las FEs. Según Best (2010), tal vez las habilidades cognitivas adquiridas durante estos juegos deportivos, que requieren la cognición compleja con el fin de cooperar con los compañeros de equipo, anticipar su comportamiento y el de los oponentes, emplear estrategias, y adaptarse a las demandas siempre cambiantes de la actividad, se transfieran a las tareas que requieren FEs.

\section{El tipo de instrucción (intencional-incidental)}

Además de la entropía de la tarea como potenciador del desarrollo de las FEs y de la creatividad táctica hay que tener en cuenta el tipo de instrucción que el deportista recibe durante su desempeño en el ejercicio. Algunas investigaciones han permitido encontrar una interferencia negativa entre las estrategias de enseńanza utilizadas para el entrenamiento de lo que hemos denominado "inteligencia táctica" y las que se utilizan para la "creatividad táctica". Para la mejora de la primera se han definido paradigmas como el de "práctica deliberada" en el cual el jugador practica en situaciones estructuradas y definidas (Côté, Baker \& Abernethy, 2003). Algunas investigaciones han encontrado que aquellos jugadores que habían sido formados durante más tiempo en una práctica deliberada y estructurada terminaron siendo menos creativos (Memmert, Baker \& Bertsch, 2010). En cambio, son varios los estudios que encontraron que la práctica sin intervención del entrenador, solo mediante juegos desestructurados y lúdicos, lo que se ha denominado el "juego deliberado" (Côté et al., 2003), tuvo una influencia positiva en el desarrollo de la creatividad táctica (Bell-Walker \& Williams, 2008; Memmert et al., 2010; Greco, Memmert \& Morales, 2010).

Esta desventaja de la práctica deliberada también se ha visto reforzada en estudios que demuestran que una instrucción intencional, en la que el entrenador ofrece información verbal de las claves atencionales relevantes para la toma de decisión, dificulta la generalización del aprendizaje de claves similares, pero no idénticas, a las utilizadas intencionadamente (Perales et al., 2011). En este sentido, Memmert y Furley (2007) descubrieron que estar sometido a información explícita de las reglas de acción tenía un efecto negativo en la capacidad de ver a un jugador desmarcado de manera inesperada.

Todo ello apoya nuestra hipótesis de que, a medida que en los deportes de interacción se utilicen metodologías de entrenamiento que adapten el nivel de entropía de la tarea al jugador, de forma que se enfrente constantemente a nuevos retos y situaciones inesperadas, y en las que el tipo de instrucción incidental utilizado promueva aprendizaje implícitos, evitando ofrecer información sobre las claves de decisión, mayor será su capacidad adaptativa, gracias a la mejora de 
sus capacidades cognitivas como las funciones ejecutivas. Por esta misma razón, mejor será su rendimiento futuro en el juego y/o menor el tiempo necesario para alcanzar su nivel de pericia más elevado.

Por otro lado, es importante valorar el efecto que el aporte de información o de resultados puede ejercer sobre la capacidad del jugador para tomar iniciativas y asumir decisiones que puedan resultar impredecibles para los contrarios. Desde los primeros trabajos de Smith y Smoll (1977) hasta la actualidad, la investigación en torno al análisis conductual de los entrenadores ha permitido, por un lado conocer la influencia de los diferentes tipos de intervención sobre variables tan determinantes para el deportista como el nivel de autoeficacia, motivación, satisfacción, disfrute o, en el sentido opuesto, el de estrés y, por otro, establecer pautas adecuadas para aportar conocimiento de resultados eficaz. Efectivamente, ha sido ampliamente demostrado que el comportamiento del entrenador influye de forma determinante sobre estas variables (Hepler \& Chase, 2008) y, dependiendo de las características o tipo de intervención, contribuir positiva o negativamente. Al mismo tiempo, sabemos que algunas de estas variables condicionan los resultados deportivos. Por ejemplo, es bien conocida la relación positiva entre la percepción de eficacia y el rendimiento deportivo (Feltz \& Lirgg, 2001; Woodman \& Hardy, 2003) e igualmente la negativa entre los niveles de ansiedad y rendimiento deportivo (Woodman \& Hardy, 2003).

Bandura (1977) identificó en su teoría cuatro fuentes de influencia que contribuyen a la percepción de eficacia personal en un dominio dado: consecución de metas pasadas, la experiencia vicaria, la persuasión verbal y los estados fisiológicos. De ellas cobran especial relevancia, para lo que nos ocupa, la primera y tercera de ellas. La sensación de haber conseguido éxito en las metas planteadas no sólo depende de la evaluación personal del deportista, por el contrario está enormemente condicionada por la que realiza el entrenador de su actuación. Ante un mismo resultado, el análisis del entrenador puede contribuir a la forma en que lo interprete el deportista. Las conductas creativas implican asumir un cierto riesgo en la decisión, al intentar realizar acciones que resulten difíciles de anticipar por el contrario. Durante el proceso de adquisición de habilidades, de entrenamiento de la capacidad decisional, el jugador de un deporte de equipo necesita ensayar acciones que, siendo adecuadas en el contexto, no siempre terminan con éxito. El refuerzo del entrenador en estas circunstancias resulta vital para consolidar la conducta al modificar el valor del resultado, dando más importancia al proceso. Esta reorientación de la valencia emocional de la experiencia ayuda al deportista a consolidar el hábito de tomar iniciativas y mejora su autoeficacia. Sin embargo, esta influencia puede ser contraria si el feedback habitual del entrenador se centra sobre el resultado negativo de la acción y no sobre la adecuación de la respuesta en el contexto de juego.
Desgraciadamente, este comportamiento puede conducir a inhibir el comportamiento deseable del jugador.

Según Weinberg y Gould (2011) cuando los deportistas cometen errores se sienten frustrados y, a menudo, sobreexcitados y ansiosos. Esto conduce a cambios improductivos del foco atencional, a incrementar la tensión muscular y a un deterioro del rendimiento. Para estos autores, es esencial que el entrenador enseñe a los deportistas a ver los errores como un medio para aprender de la experiencia, lo que le exige el valorar positivamente la toma de iniciativa cuando la decisión sea acertada aunque no lo sea el resultado.

\section{Aplicaciones prácticas}

De todo lo anterior podemos extraer algunas conclusiones que nos sirvan de guía para el diseño de escenarios de aprendizaje orientados a la mejora de la creatividad táctica, e inevitablemente vinculadas al desarrollo de las funciones ejecutivas. Con respecto al diseño de tareas proponemos:

- La tarea debe contemplar un cierto nivel de incertidumbre. Para ello podemos utilizar diferentes estrategias (Cárdenas et al, 2015):

o Manipular el número de alternativas que tiene el jugador. Esto se puede conseguir controlando el grado de libertad de los adversarios o estableciendo normas de comportamiento que regulen las opciones de juego.

o Manipular de forma aleatoria la aparición de los estímulos a atender.

- Aumento progresivo de la complejidad perceptiva de la tarea:

o Aumentar el número de estímulos presentes a los que atender.

o Aumentar la velocidad de los estímulos.

o Disminuir el tiempo de exposición al estímulo (por ejemplo, la doble tarea hace que tengas que repartir el tiempo entre ambas).

o Reducir el tiempo disponible para resolver la situación de juego.

o Generar situaciones de asimetría numérica desfavorable (jugar en inferioridad).

o Generar situaciones de asimetría física o corporal (los más altos juegan contra los más bajos; los más rápidos contra los más lentos, etc.).

o Generar situaciones de fatiga mental y física, que normalmente deterioran el rendimiento perceptivo, para provocar respuestas adaptativas de los jugadores.

- Utilizar estrategias para el desarrollo de la inhibición:

o La tarea debe contemplar la presencia de elementos distractores.

o Estímulos inesperados para que el jugador tenga 
que dejar de realizar su acción automática para dar respuesta a este nuevo estímulo.

o Cambiar el objetivo de la tarea durante su transcurso y una vez aprendida.

o Varios roles dentro de la tarea.

o Prohibición de conductas ya muy asentadas o naturales. Ej.: prohibido pasar al jugador que te ha pasado o al jugador más cercano o contiguo.

- Utilizar estrategias para el desarrollo de la actualización de la memoria de trabajo:

o Tareas en las que el jugador deba estar cambiando constantemente el foco atencional para adecuar su acción a dos o más estímulos simultáneos. Ej.: el uso de más de un balón en una tarea, el intercambio de roles con varios equipos con colores diferentes en función de una norma dada o de la recuperación de la posesión.

- Tareas en la que el jugador debe actualizar la información que debe recordar según normas de comportamiento. Por ejemplo, está prohibido pasar al jugador del que has recibido el pase.

- Utilizar estrategias que estimulen el desarrollo de la flexibilidad cognitiva.

o Obligar a cambiar el tipo de solución a un problema motor dado o premiar el hecho de hacerlo. Por ejemplo, el entrenador muestra mediante un código numérico el tipo de finalización para el equipo atacante, sin que lo vea el equipo defensor (se coloca a la espalda de la defensa). o Sobre una situación de juego determinada cambiar el objetivo de consecución.

o Establecer rotaciones de los roles dentro de una tarea combinando estrategias por turno y por consecución de objetivos.

o Introducir consignas que obliguen al cambio de uso de formas colectivas de juego tanto en ataque como defensa, dependiendo de las circunstancias de juego (si se consigue canasta o no; si se produce saque de banda, si estamos por encima o debajo en el marcador, etc.).

Con respecto a las estrategias de intervención del entrenador creemos interesante tener en cuenta las siguientes propuestas:

- Uso de estrategias incidentales. El entrenador no hará coincidir el objetivo de la tarea con el objetivo motor.

- Evitar corregir al jugador utilizando un feedback prescriptivo y dirigido.

- Usar un feedback afectivo positivo cuando el jugador realice una toma de decisión adecuada. Esto va a generar al jugador una emoción positiva que podrá asociar a su toma de decisión.

- Evitar el uso de feedback negativo cuando el jugador realice una toma de decisiones adecuada, pero arriesgada, que conlleve un resultado negativo. En estos casos es aconsejable reforzar la decisión adecuada para quitarle importancia al resultado negativo.

\section{Referencias bibliográficas}

1. Abernethy, B., Baker, J., \& Côté, J. (2005). Transfer of pattern recall skills may contribute to the development of sport expertise. Applied Cognitive Psychology, 19, 705-718.

2. Alarcón, F., \& Verdú, A. (2012). La toma de decisiones de jugadores de baloncesto amateurs en condiciones de alta complejidad perceptiva. En R. Escobar \& A. Sánchez, (Eds.), Libro de actas. VII Congreso Internacional de la Asociación Española Ciencias del Deporte (pp. 360). Granada, España: Universidad de Granada.

3. Alves, H., Voss, M. W., Boot, W. R., Deslandes, A., Cossich, V., Salles, J. I., \& Kramer, A. F. (2013). Perceptual-cognitive expertise in elite volleyball players. Frontiers in Psychology, 4, 36.

4. Bandura, A. (1977). Self-efficacy: toward a unifying theory of behavioral change. Psychological review, 84(2), 191.

5. Bell-Walker, J., \& Williams, A.M. (2008). The effect of memory recall on perceptual-cognitive skill in elite soccer: Development of long term working memory. En T. Reilly, F. Korkusuz \& E. Ergen (Eds.), Science and football VI (pp. 340-343). Londres, Reino Unido: Taylor y Francis.

6. Ben-Soussan, T. D., Berkovich-Ohana, A., Piervincenzi, C., Glicksohn, J., \& Carducci, F. (2015). Embodied cognitive flexibility and neuroplasticity following Quadrato Motor Training. Front. Psychol., 6, 1021. doi: 10.3389/fpsyg. 2015.01021

7. Best, J. R. (2010). Effects of physical activity on children's executive function: Contributions of experimental research on aerobic exercise. Developmental Review, 30(4), 331-351.
8. Brady F. (2008). The contextual interference effect and sport skills. Perceptual and Motor Skills, 106, 461-472.

9. Carey, J. R., Bhatt, E., \& Nagpal, A. (2005). Neuroplasticity promoted by task complexity. Exercise and Sport Sciences Reviews, 33, 24-31.

10. Cárdenas, D., \& Alarcón, F. (2010). Conocer el juego en baloncesto para jugar de forma inteligente. Revista Wanceulen EF Digital, 6, 51-72.

11. Cárdenas, D., Conde-González, J., \& Perales, J. C. (2015). El papel de la carga mental en la planificación del entrenamiento deportivo. Revista de psicología del deporte, 24(1), 91-100.

12. Cárdenas, D. \& Torre, E. (2005). El desarrollo de la creatividad en el deporte. En J. Giménez \& Sáenz-Lopez (Eds.),Educar a través del deporte. Huelva, España: Servicio de publicaciones de la Universidad de Huelva.

13. Chaddock, L., Neider, M. B., Voss, M. W., Gaspar, J. G., \& Kramer, A. F. (2011). Do athletes excel at everyday tasks? Medicine and Science in Sports y Exercise, 43, 1920-1926.

14. Côté, J., Baker, J., \& Abernethy, B. (2003). From play to practice: a developmental framework for the acquisition of expertise in team sports. En J. L. Starkes \& K. A. Ericsson (Eds.), Recent advances in research on sport expertise (pp. 89-110). Champaign, IL: Human Kinetics.

15. Cross, E. S., Schmitt, P. J.,\& Grafton, S. T. (2007). Neural substrates of contextual interference during motor learning support a model of active preparation. Journal of Cognitive Neuroscience, 19, 1854-1871.

16. Faubert, J. (2013). Professional athletes have extraordinary skills for 
rapidly learning complex and neutral dynamic visual scenes. Scientific Reports, 3, 1154.

17. Feltz, D. L., \& Lirgg, C. D. (2001). Self-efficacy beliefs of athletes, teams, and coaches. Handbook of sport psychology, 2(2001), 340-361.

18. Furley, P., Memmert, D., \& Heller, C. (2010). The dark side of visual awareness in sport: Inattentional blindness in a real-world basketball task. Attention, Perception y Psychophysics, 72, 1327-1337. PubMed doi:10.3758/APP.72.5.1327

19. Greco, P., Memmert, D., \& Morales, J. C. P. (2010). The effect of deliberate play on tactical performance in basketball. Perceptual and Motor Skills, 110, 849-856.

20. Guilford, J. P. (1967).The nature of human intelligence. New York: McGraw Hill.

21. Hepler, T. J., \& Chase, M. A. (2008). Relationship between decisionmaking self-efficacy, task self-efficacy, and the performance of a sport skill. Journal of Sports Sciences, 26(6), 603-610.

22. Hogarth, R. M. (2001). Educating Intuition. Chicago, IL: The University of Chicago Press.

23. Lezak, M. D. (1982). The problem of assessing executive functions. Int J Psychol, 17, 281-97.

24. Mangine, G. T., Hoffman, J. R., Wells, A. J., Gonzalez, A. M., Rogowski, J. P., Townsend, J. R., Jajtner, A. R., Beyer, K. S., Bohner, J. D., Pruna, G. J., Fragala, M. S., \& Stout, J. R. (2014). Visual Tracking Speed Is Related to Basketball-Specific Measures of Performance in NBA Players. Journal of strength and conditioning research, 28, 2406-2414.

25. Marek, T., Noworol, C., \& Karwowski, W. (1988). Mental fatigue at work and pain perception. Work and Stress, 2(2), 133-137.

26. Martín, I., Chirosa, L. J., Reigal, R. E., Hernández, A., Juárez, R., \& Guisado, R. (2014). Efectos de la actividad física sobre las funciones ejecutivas en una muestra de adolescentes. Anales de Psicologia, 31(3), 962-971.

27. Martins, N., \& Gotuzo, A. (2015). Is it possible to promote executive functions in preschoolers? A case study in Brazil. International Journal of Child Care and Education Policy, 9(6). doi:10.1186/s40723-015-00102

28. Memmert, D. (2014). Tactical creativity in team sports. Research in Physical Education, Sport and Health, 3(1), 13-18.

29. Memmert, D., \& Furley, P. (2007). "I spy with my little eye!”: Breadth of attention, inattentional blindness, and tactical decision making in team sports. Journal of Sport y Exercise Psychology, 29, 365-381.

30. Memmert, D., \& Roth, K. (2007). The effects of non-specific and specific concepts on tactical creativity in team ball sports. Journal of Sport Science, 25, 1423-1432.

31. Memmert, D., Baker, J., \& Bertsch, C. (2010). Play and Practice in the Development of Sport-Specific Creativity in Team Ball Sports. High Ability Studies, 21, 3-18.

32. Miyake, A., Friedman, N. P., Emerson, M. J., Witzki, A. H., Howerter, A., \& Wager, T. D. (2000). The unity and diversity of executive functions and their contributions to complex frontal lobe tasks: a latent variable analysis. Cognition Psychology, 41, 49-100.

33. North, J. S., \& Williams, A. M. (2008). Identifying the critical time period for information extraction when recognizing sequences of play. Research Quarterly for Exercise and Sport, 79, 268-273.

34. Park, D. C.,\& Shaw, R. J. (1992). Effect of environmental support on implicit and explicit memory in younger and older adults. Psychol Aging, 7, 632-642.
35. Perales, J.C., Cárdenas, D., Piñar, M.I., Sánchez, G., \& Courel, J. (2011). El efecto diferencial de la instrucción incidental e intencional en el aprendizaje de las condiciones para la decisión de tiro en baloncesto. Revista Psicología del Deporte, 20(2), 729-745.

36. Pesce, C., Crova, C., Marchetti, R., Struzzolino, I., Masci, I., Vannozzi, G., \& Forte, R. (2013). Searching for cognitively optimal challenge point in physical activity for children with typical and atypical motor development. Mental Health and Physical Activity, 6(3), 172-180. doi:10.1016/j.mhpa.2013.07.001

37. Romeas, T., Guldner, A.,\& Faubert, J. (2015). 3D-Multiple Object Tracking task performance improves passing decision-making accuracy in soccer players. Psychology of Sport y Exercise. doi: 10.1016/j.psychsport.2015.06.002.

38. Runco, M. A. (2007). Creativity - theories and themes: research, development, and practice. Burlington, MA: Elsevier.

39. Smeeton, N. J., Ward, P., \& Williams, A. M. (2004). Do pattern recognition skills transfer across sports? A preliminary analysis. Journal Sports Sciences, 22, 205-213.

40. Smith, R.E., \& Smoll, F.L. (1977). Coach mediated team building in youth sports. Journal of Applied Sport Psychology, 9, 114-132.

41. Sternberg, R. J., \& Lubart, T. I. (1999). The Concept of Creativity: Prospects and Paradigms. En R. J. Sternberg (Ed.), Handbook of creativity (pp. 3-15). Cambridge: Cambridge University Press.

42. Tirapu-Ustárroz, J., García-Molina, A., Luna-Lario, P., Verdejo-García, A., \& Ríos-Lago, M. (2012). Corteza prefrontal, funciones ejecutivas y regulación de la conducta. En J. Tirapu-Ustárroz, A. García-Molina, M. Ríos-Lago \& A. Ardila-Ardila (Eds.), Neuropsicología de la corteza prefrontaly las funciones ejecutivas (pp. 87-120) Barcelona, España: Viguera.

43. Vaeyens, R., Lenoir, M., Williams, A. M., \& Philippaerts, R. M. (2007). Mechanisms underpinning successful decision making in skilled youth soccer players: An analysis of visual search Behaviors. Journal of Motor Behavior, 39, 395-408.

44. Van Praag, H., Christie, B. R., Sejnowski, T. J., \& Gage, F. H. (1999), Running enhances neurogenesis, learning, and long-term potentiation in mice. Proceedings of the National Academy of Sciences, 96, 1342713431.

45. Verburgh, T., Scherder, E., Van Lange, P., \& Oosterlaan, J. (2014). Executive Functioning in Highly Talented Soccer Players. Plos One, 9(3).

46. Vestberg, T., Gustafson, R., Maurex, L., Ingvar, M., \& Petrovic, P. (2012). Executive Functions Predicts the Success of Top-Soccer Players. Plos One, 7(4).

47. Weinberg, R. S., \& Gould, D. (2011). Foundations of Sport and Exercise Psychology (5a ed.). Champaign, IL: Human Kinetics.

48. Williams, A. M., Hodges, N. J., North, J. S., \& Barton, G. (2006). Perceiving patterns of play in dynamic sport tasks: Investigating the essential information underlying skilled performance. Perception, 35, 317-332.

49. Williams, M. (Ed.) (2013). Science and Soccer: Developing Elite Performance. Abingdon: Routledge.

50. Williams, M. A. (2000). Perceptual skill in soccer: implications for talent identification and development. Journal Sports Sciences, 18, 737 750 .

51. Woodman, T., \& Hardy, L. (2003). The relative impact of cognitive anxiety and self-confidence upon sport performance: A meta-analysis. Journal of sports sciences, 21(6), 443-457. 